Iván Fernando Romero-Velásquez; Darwin Gabriel García-Herrera; Cristian Andrés Erazo-Álvarez Juan Carlos Erazo-Álvarez

http://dx.doi.org/10.35381/e.k.v3i1.1016

\title{
Podcast como recurso didáctico para desarrollar habilidades comunicativas
}

Podcast didactic resource to develop communicative skills

\author{
Iván Fernando Romero-Velásquez \\ ivan.romero.38@est.ucacue.edu.ec \\ Universidad Católica de Cuenca, Cuenca \\ Ecuador \\ https://orcid.org/0000-0001-8419-9595 \\ Darwin Gabriel García-Herrera \\ dggarciah@ucacue.edu.ec \\ Universidad Católica de Cuenca, Azogues \\ Ecuador \\ https://orcid.org/0000-0001-6813-8100 \\ Cristian Andrés Erazo-Álvarez \\ cristianerazo@ucacue.edu.ec \\ Universidad Católica de Cuenca, Cuenca \\ Ecuador \\ https://orcid.org/0000-0001-8746-4788 \\ Juan Carlos Erazo-Álvarez \\ jcerazo@ucacue.edu.ec \\ Universidad Católica de Cuenca, Cuenca \\ Ecuador \\ https://orcid.org/0000-0001-6480-2270
}

Recepción: 10 agosto 2020

Revisado: 25 septiembre 2020

Aprobación: 15 octubre 2020

Publicación: 01 noviembre 2020 


\title{
RESUMEN
}

El objetivo de la investigación se basó en analizar el Podcast como recurso didáctico para desarrollar habilidades comunicativas en estudiantes de nivel de bachillerato de la Unidad Educativa Particular Liceo Americano Católico. De tipo descriptiva con diseño no experimental de cohorte transversal, siendo la muestra poblacional de 48 estudiantes. EI cruce de variables se obtiene una asignación bilateral de 0.000 este resultado demuestra la relación entre las variables analizadas evidenciando que el podcast es útil para desarrollar experiencias de aprendizaje por lo concerniente la hipótesis es válida. El podcast es útil para el proceso de enseñanza-aprendizaje puesto que su diseño es el mecanismo que permite desarrollar destrezas y competencias interdisciplinarias en cada uno de los usuarios.

Descriptores: Comunicación móvil; telecomunicación; relaciones interpersonales. (Palabras tomadas del Tesauro UNESCO).

\begin{abstract}
The objective of the research was based on analyzing the Podcast as a didactic resource to develop communication skills in high school students of the Private Educational Unit Liceo Americano Católico. Descriptive with a non-experimental cross-sectional cohort design, the population sample being 48 students. The crossover of variables gives a bilateral assignment of 0.000 . This result demonstrates the relationship between the analyzed variables, showing that the podcast is useful for developing learning experiences, as far as the hypothesis is valid. The podcast is useful for the teachinglearning process since its design is the mechanism that allows developing interdisciplinary skills and competencies in each of the users.
\end{abstract}

Descriptors: Mobile communication; telecommunications; interpersonal relations. (Words taken from the UNESCO Thesaurus). 


\section{INTRODUCCIÓN}

Comunicar es una habilidad, mientas tanto que; trasmitir un mensaje constituye sintonizar con los interlocutores con el objetivo de informar; cada ser humano posee su forma de expresar y vivir sus experiencias, por tal razón; saber comunicar es un componente necesario para que las personas puedan relacionarse entre sí, las destrezas de comunicación que ostentan el hombre establecen el éxito de las relaciones interpersonales; Dicho en otras palabras; es importante saber comunicarse de manera correcta; en la escuela, el trabajo, con la familia, los amigos, en general con todas las personas.

Para lograr un feedback eficaz es necesario dominar la lectoescritura, puesto que; leer y escribir son las herramientas base para la comunicación. En efecto, leer ayuda al desarrollo intelectual del sujeto, brinda el discernimiento necesario para dialogar críticamente, pero también sirve de elemento cognitivo el cual permite comprender mejor la realidad de la sociedad, pero se debe considerar que; no siempre las personas se comunican de forma oral, por ello, es trascendental dominar la destreza de la comunicación escrita.

Conseguir que la lectoescritura se convierta en un hábito cotidiano en la sociedad es una tarea completa, las personas consideran que leer o escribir es un trabajo fastidioso y aburrido. No obstante; leer no es solo cumplir con una necesidad, es aprender vocabulario, es desarrollar la memoria, el pensamiento y la creatividad. La lectura en todos los niveles académicos es necesaria para el óptimo aprovechamiento de los alumnos, así como para el mejoramiento de su desempeño intelectual y cognitivo en todos los aspectos de la vida. (Flores, 2016)

Pero; ¿Qué se entiende por el termino lectura y escritura? La primera hace referencia a la edificación de un significado, en otras palabras; se refiere a la decodificación de los sonidos, mientras tanto que; la escritura representa la voz del pensamiento por medio de 
las letras, cuando el individuo no sabe leer correctamente, éste no decodifica de manera adecuada el mensaje, como resultado obtendrá una mala interpretación de la información, un patrón similar se presenta cuando no saben escribir bien.

La lectoescritura al igual que hablar y escuchar es una herramienta que funciona como mediadora entre el emisor y el receptor, quienes son participes del proceso de comunicación, y a su vez se convierten en elementos necesarios para desarrollar conocimientos significativos en el sujeto. (Monsalve-Upegui, et al., 2011).

En Latinoamérica los índices de lectura son relativamente bajos, en el informe emitido por el Centro Regional para el Fomento del Libro en América Latina y el Caribe (Cerlalc), publicado en el año 2012. En el Ecuador, los habitantes leen la mitad de un libro en el año, la publicación revela que el país posee uno de los índices más bajos en relación a los 5,4 libros que leen los chilenos al año. El Ecuador posee un $43 \%$ de sociedad lectora, frente a un $77 \%$ en Colombia.

En el entorno educativo muchos docentes tradicionalistas se enfocan en la apariencia de la redacción, es lamentable, en escasas ocasiones, se enfoca en el contenido o en el mensaje escrito. Por ello es necesario incentivar en las aulas de clase a desarrollar en los estudiantes destrezas de lectura y escritura por medio de recursos didácticos nuevos, los mismos que permitan a los educandos desarrollar habilidades para la vida y a la vez aprendizaje significativo. En este contexto, el podcast se presenta como un recurso didáctico que promueve las capacidades de lectura y escritura, esta herramienta bajo una estrategia pedagógica se convierte en un elemento esencial en el proceso de enseñanza - aprendizaje, ya que permite retroalimentar, corregir y potencializar las habilidades comunicación de manera sincrónica como asincrónica.

Las tecnologías de la comunicación e información se han en el convertido componente de interacción, que no solo permite establecer procesos de comunicación, sino también, estimula al: diálogo, a la reflexión, al análisis de la coyuntura social. Reflexionemos ahora; sobre el contraste entre el uso del podcast comercial con educativo; para unos este 
recurso es un mecanismo comercial, para otros el podcast es un medio que permiten la reflexión y la interiorización de conocimientos.

Como recurso didáctico el podcast permite un fácil acceso a la información, permitiendo así una difusión y comunicación de contenidos pedagógicos y por supuesto genera un proceso de manejo y aprendizaje de herramientas y material virtual de calidad y de fácil acceso. (Aldana-Talero, 2012) Por ello la presente investigación tiene como objetivo describir al Podcast como Recurso Didáctico para desarrollar Habilidades Comunicativas. En este sentido, el objetivo de la investigación se basó en analizar el Podcast como recurso didáctico para desarrollar habilidades comunicativas en estudiantes de nivel de bachillerato de la Unidad Educativa Particular Liceo Americano Católico.

\section{Referencial teórico}

Con la intención de respaldar la propuesta de estudio, es necesario investigar trabajos afines, los mismos que fortalecerán el objetivo planteado. La investigación teórica encierra dos variables: las habilidades comunicativas y el podcast como recurso didáctico. En primer lugar, es necesario señalar que las habilidades de comunicación permiten al ser humano relacionarse con los demás por medio de la palabra oral, escrita, simbólica y mímica; todos estos códigos permiten entender el mensaje que otro individuo está transmitiendo, por ello es primordial desarrollar habilidades de comunicación para expresar de manera eficaz; emociones, pensamientos, etc. Todo esto parece confirmar que; gracias a la comunicación, la sociedad ostenta un gran desarrollo social, en otras palabras, el evento comunicacional constituye la base primordial de la vida social y es la herramienta más poderosa para cambiar a la humanidad.

Exploremos un poco la idea; el lenguaje, aparte de ser un mecanismo de comunicación básico para la socialización, es también un medio usado para expresar o comunicar un contenido relacionado con el pensamiento, Para comprender mejor (Gómez, 2016) afirma; el pensar y el hablar forman parte de una unidad inseparable: no se puede 
considerar como una simple manera de comunicación sino como un método importante para el desarrollo y visión que tiene el hablante hacia el mundo. Es posible que, los estudiantes se les dificulte expresarse de forma oral o escrita, sobre todo con los docentes al momento de interactuar en clase, en una exposición, o en un diálogo, las faltas del manejo de habilidades expresivas impiden una comunicación asertiva, toda esta problemática se debe a la falta del incentivo tanto de docentes como de alumnos a desarrollar competencias digitales para el auto aprendizaje.

En la actualidad tenemos a un gran aliado, la tecnología es el arma que nos ayuda a enfrentar y mejorar dichas debilidades de educadores y educandos, indiscutiblemente el desarrollo tecnológico ha evolucionado en cuanto al uso y la manera de interactuar en la red, en este entorno los usuarios ya no solo se conectan por medio de sus ordenadores, sino también lo hacen a través de sus dispositivos móviles, como las Tablet, los celulares incluso los relojes inteligentes, con esto los internautas acceden a las nuevas formas de visualizar, obtener y publicar contenidos. El podcast como herramienta para desarrollar habilidades de comunicación y aprendizaje de contenidos no ha sido empleado de manera habitual y efectiva en las sesiones de clase.

\section{Habilidades Comunicativas}

El ser humano desde que nace interacciona en su hábitat, a medida que crece, la habilidad de comunicarse se va ampliando de manera lógica e intelectual, es decir; con el paso del tiempo su expresión lingüística va creciendo, por consiguiente, las habilidades comunicativas se refieren a la capacidad que el individuo tiene para comunicarse con los demás. En ocasiones, el diálogo se dificulta, puesto que el individuo no encuentra los términos adecuados para expresar sus pensamientos; es evidente que; al no trasmitir certeramente el mensaje, la información se descontextualiza, se desnaturaliza y por ende el acto comunicativo se ve afectado. Para que esta condición no suceda es fundamental desarrollar habilidades como las de; escuchar, hablar, escribir y leer, dado que son 
elementos ineludibles en el ser humano.

La comunicación es una condición primordial de la existencia del hombre, por lo que no sería posible el desarrollo de la sociedad sin tenerla en cuenta, es un fenómeno que implica relaciones humanas de socialización e intercambio de mensajes durante su participación en las actividades que realiza cotidianamente. (Cevallos-Mieles, 2016). Cabe considerar por otra parte que; cuando el ser humano es parte de un entorno pobre en léxico, manifestará numerosos problemas para entender y por ende adquirir conocimiento necesario para desenvolverse socialmente.

El sujeto que apenas interpreta lo que escribe o habla sin pertinencia, casi no lee o lo hace con suma dificultad y escribe de manera defectuosa, tendrá dificultad en casi todos los ámbitos de su vida personal, laboral y social. Hasta para escuchar la radio o ver la televisión se requiere cierto grado de competencia lingüística, pues vivir es convivir y esta convivencia se basa en la comunicación interpersonal para estar informado y sentirse incluido dentro del grupo. (Reyzábal, 2012)

Bajo este contexto es vital que los seres humanos desde muy pequeños desarrollen habilidades en cuanto al manejo del lenguaje, los centros educativos están llamados a estimular estas destrezas en todas las áreas del conocimiento. La educación del nivel básico cumple un papel primordial en el proceso de aprendizaje de habilidades lingüísticas gracias a un bagaje de prácticas de lectoescritura, las cuales permiten optimizar la compresión del niño y por ende ayudan a concebir sus primeras nociones de trasmitir un mensaje.

Uno de los componentes esenciales de la humanidad es la escuela, como institución social está comisionada a ejecutar procesos de alfabetización y formación para el uso de la lectura y escritura, con la intención de ponerla al servicio de la producción y reproducción del sistema social. (Miranda-Calderón, 2011). 


\section{La falta de una buena comunicación en los salones de clase}

Los individuos requieren estar integrados para sentirse parte de un grupo social; en el hogar, los amigos, los compañeros de clase, etc., en efecto la interacción es el lugar donde aprendemos, cuando el ser humano experimenta otras realidades sociales, promueven diferentes tipos de comunicación, esto involucra, establecer y generar relaciones afectivas, que generen bienestar, basadas en la colaboración y cooperación. Como la expresa (Cubero-Venegas, 2004), por intermedio de la comunicación, en gran parte llegamos a ser lo que somos; por medio de la comunicación la sociedad aprende; una comunicación distorsionada ocasiona problemas de relaciones humanas, tales inconvenientes se solucionan por medio de una comunicación asertiva.

La interacción es el lugar donde aprendemos, es decir cuando el ser humano experimenta otras realidades sociales, promueven diferentes tipos de comunicación, lo que involucra, establecer y generar relaciones efectivas, que generen bienestar, basadas en la colaboración y cooperación. El acto comunicativo contiene información que es percibida por otra persona, sin importar la condición del mensaje, podrá ser o no verbal, lo que verdaderamente resalta dentro del proceso comunicativo es que tenga consigo un mensaje.

La comunicación asertiva dentro del contexto educativo se la definen como un recurso ineludible para promover el aprendizaje en los educandos, por ello es necesario que el docente establezca estrategias comunicacionales que desarrollen una conducta asertiva durante la jornada escolar y que vigoricen las capacidades individuales y del grupo. (Cañas-Betancur \& Hernández-Sánchez, 2019).

\section{El Uso del Podcast para desarrollar habilidades de comunicación}

La comercialización que se relaciona con el audio y vídeo ha revolucionado el mercado digital, este fenómeno ocurre debido a la conducta de los consumidores, los mismos que están en una búsqueda constante de innovación y exigencia de contenidos. Bajo este 
principio las empresas han desarrollado nuevos métodos para la comercialización de archivos multimedia, uno de ellos es el podcast, el cual se origina en primera instancia por medio de la palabra "iPod", mismo que se relaciona con un contenido portátil, mientras que el término "Broadcast", se refiere a la transmisión del contenido, el cual persigue el mismo patrón de la radiodifusión, la característica que difiere al podcast de un programa de radio, reside en la posibilidad de descargar los archivos sonoros para reproducirlos incluso de modo offline.

Ahora veamos, pero ¿Qué es el Podcast? Básicamente son capítulos o episodios grabados en audio que son difundidos de manera online, este recurso se vuelve versátil gracias a su diseño el cual se adapta a diferentes formatos como la entrevista, los debates, las grabaciones individuales, o simplemente el presentador narra un tema de actualidad y de interés social. El podcast consigue aplicarse en variados proyectos sonoros, esta herramienta permite; buscar escuchar y descargar asincrónicamente el contenido a través de un sin número de plataformas web; el formato del podcast tiene contenidos llamativos y muy variados, el soporte online de esta herramienta concede ampliar el entorno de cobertura de los contenidos puesto que; son ajustables a programas de entrenamiento y académicos. (Quintana-Guerrero, et al., 2017)

El podcast es un recurso que permite desarrollar habilidades de comunicación, actualmente el uso de esta herramienta está en aumento, gracias a su fácil producción, distribución. El desarrollo tecnológico ha viabilizado un cambio en la producción y en la distribución de información, estos se han adaptado especialmente en la recepción de los contenidos con terminales conectados a Internet, los cuales han equilibrado las posibles diferencias en las experiencias digitales. (Moreno-Cazalla, 2017).

En el ámbito educativo el podcast constituye un elemento formidable para el salón de clase, el elemento clave del podcast para inmiscuirse en los procesos de enseñanzaaprendizaje reside en la capacidad de desarrollar hábitos de escritura y lectura, permite también que el aprendizaje sea autónomo, en otras palabras, los contenidos educativos 
pueden ser aprovechados en cualquier parte del mundo, gracias a las bondades de la internet.

Las tecnologías digitales juegan un rol predominante en el aprendizaje de contenidos, en parte provee el acceso a una extensa variedad de materiales educativos y, por otra parte, fomenta nuevas formas de aprendizaje unidireccional cediendo el acceso a la información, transmisión e intercambio de saberes por medio de múltiples medios de comunicación. (Boulahrouz-Lahmidi, et al., 2019). El podcast como recurso didáctico es útil para que el estudiante amplíe las habilidades de investigación, proporcionado así un aprendizaje autónomo; trasladar este proceso personal conlleva al educando un esfuerzo de asimilación y optimización del saber, creando así un producto coherente y eficaz, mismo que le permitirá desarrollará competencias digitales. La implementación de un podcast demanda un proceso de reflexión y organización proporcionadas, que ha de concluir en una valoración potencialmente proyectada en la distribución previa que nos sirva para mejorar los futuros procesos. (Solano-Fernández \& Sanchez-Vera, 2010).

\section{METODOLOGÍA}

La investigación es de tipo descriptiva con diseño no experimental de cohorte transversal, siendo la muestra poblacional de 48 estudiantes de nivel de bachillerato de la Unidad Educativa Particular Liceo Americano Católico, ubicada en la zona urbana la ciudad de Cuenca en la Provincia del Azuay. Se empleó como tecnica de recolección de datos, la encuesta online y muestreo aleatorio, mientras que el instrumento fue un cuestionario de opción multiple, validado por juicio de expertos y prueba piloto para el análisis de fiabilidad de Alfa de Cronbach, la cual arrojó como resultados de 0,943 Los datos recopilados fueron analizados en función de la estadistica descriptiva y chi cuadrado de Pearson. 
Iván Fernando Romero-Velásquez; Darwin Gabriel García-Herrera; Cristian Andrés Erazo-Álvarez Juan Carlos Erazo-Álvarez

\section{RESULTADOS}

En este apartado se describen los principales resultados obtenidos en la investigación:

Tabla 1.

Análisis descriptivo variables.

\begin{tabular}{|c|c|c|c|c|c|}
\hline & \multicolumn{2}{|c|}{$\mathrm{N}$} & Media & Mediana & Moda \\
\hline & Validos & Perdidos & & & \\
\hline $\begin{array}{l}\text { Crees que el uso frecuente del podcast es útil para } \\
\text { desarrollar nuevas experiencias de aprendizaje. }\end{array}$ & 48 & 0 & 4.10 & 4.00 & 4 \\
\hline $\begin{array}{l}\text { Crees que el podcast es una herramienta que te } \\
\text { ayuda a desarrollar habilidades comunicativas }\end{array}$ & 48 & 0 & 4.13 & 4.00 & 5 \\
\hline $\begin{array}{l}\text { Consideras que el podcast posee información desde } \\
\text { el entorno académico }\end{array}$ & 48 & 0 & 3.81 & 4.00 & 4 \\
\hline $\begin{array}{l}\text { Crees que el podcast muestra información clara y } \\
\text { contundente sobre los contenidos }\end{array}$ & 48 & 0 & 4.06 & 4.00 & 4 \\
\hline
\end{tabular}

Fuente: Elaboración Propia.

Como se puede observar en la tabla 1 se obtuvieron 48 encuestados válidos, es decir el $100 \%$ de la muestra propuesta. La encuesta se enfoca en el uso del podcast para obtener nuevas experiencias de aprendizaje, también se orienta a indagar si los estudiantes consideran al podcast como una herramienta que ayuda a desarrollar habilidades de comunicación, cuestiona si consideran que la información que posee podcast es de carácter académico, a su vez consulta si creen que el podcast tiene información clara y contundente sobre los contenidos que divulga. Los valores obtenidos en la media, mediana y moda guardan relación siendo estas cifras puntos de referencia para interpretar los resultados alcanzados en la encuesta. 
EPISTEME KOINONIA

Revista Electrónica de Ciencias de la Educación, Humanidades, Artes y Bellas Artes

Año III. Vol III. N¹. Edición Especial. 2020

Hecho el depósito de Ley: FA2018000022

ISSN: 2665-0282

FUNDACIÓN KOINONIA (F.K).

Santa Ana de Coro, Venezuela

Iván Fernando Romero-Velásquez; Darwin Gabriel García-Herrera; Cristian Andrés Erazo-Álvarez Juan Carlos Erazo-Álvarez

En cuanto a la relación entre variables tenemos los siguientes resultados:

Tabla 2.

Crees que el uso frecuente del podcast es útil para desarrollar nuevas experiencias de aprendizaje. ${ }^{*}$ El podcast es útil para el aprendizaje de contenidos.

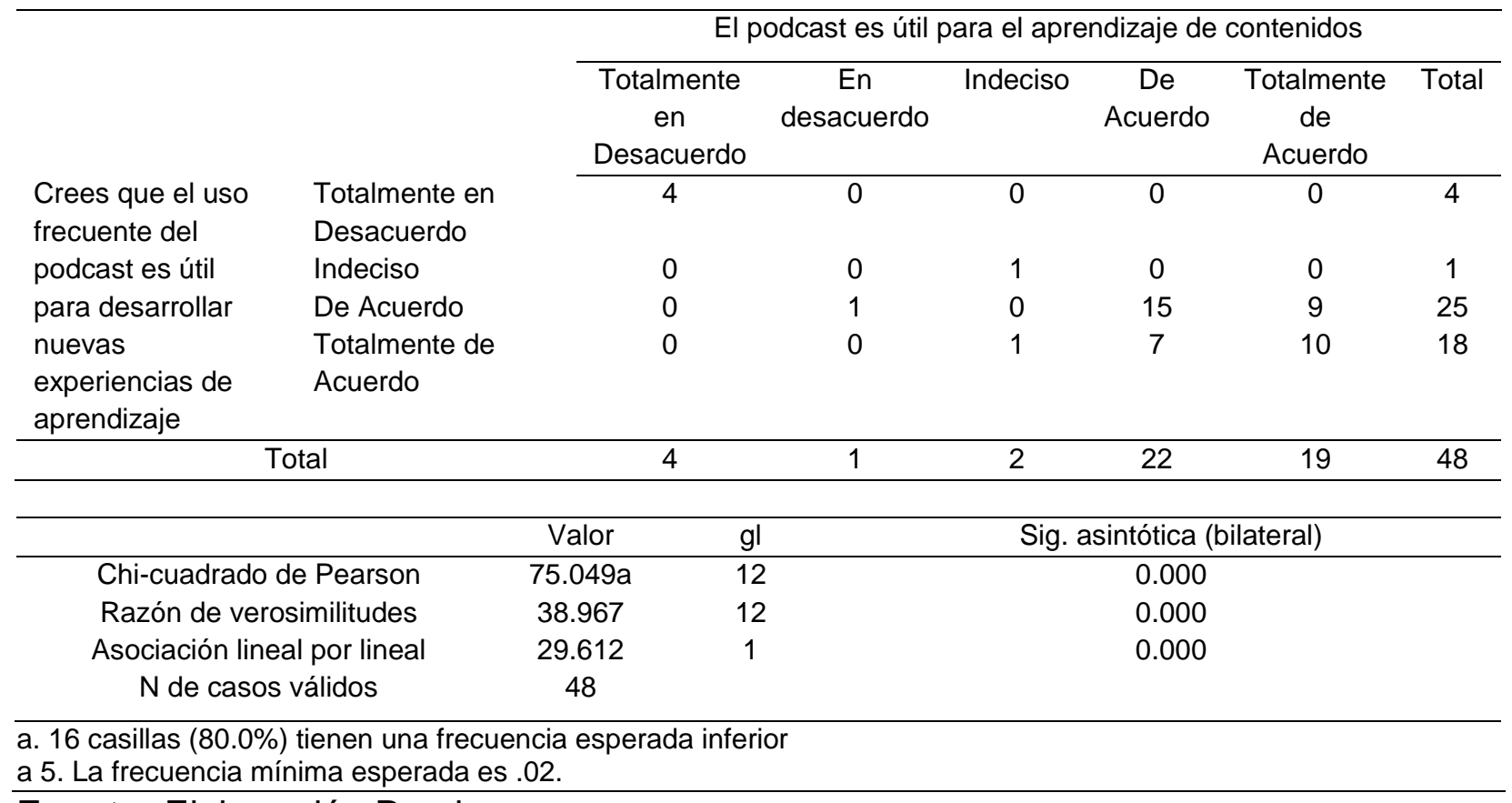

Fuente: Elaboración Propia.

Como se puede observar en la tabla 2, el cruce de variables se obtiene una asignación bilateral de 0.000 este resultado demuestra la relación entre las variables analizadas evidenciando que el podcast es útil para desarrollar experiencias de aprendizaje por lo concerniente la hipótesis es válida. 
EPISTEME KOINONIA

Revista Electrónica de Ciencias de la Educación, Humanidades, Artes y Bellas Artes

Año III. Vol III. N¹. Edición Especial. 2020

Hecho el depósito de Ley: FA2018000022

ISSN: 2665-0282

FUNDACIÓN KOINONIA (F.K).

Santa Ana de Coro, Venezuela

Iván Fernando Romero-Velásquez; Darwin Gabriel García-Herrera; Cristian Andrés Erazo-Álvarez Juan Carlos Erazo-Álvarez

\section{Tabla 3.}

Crees que el podcast es una herramienta que te ayuda a desarrollar habilidades comunicativas * El podcast motiva a desarrollar hábitos de lectura.

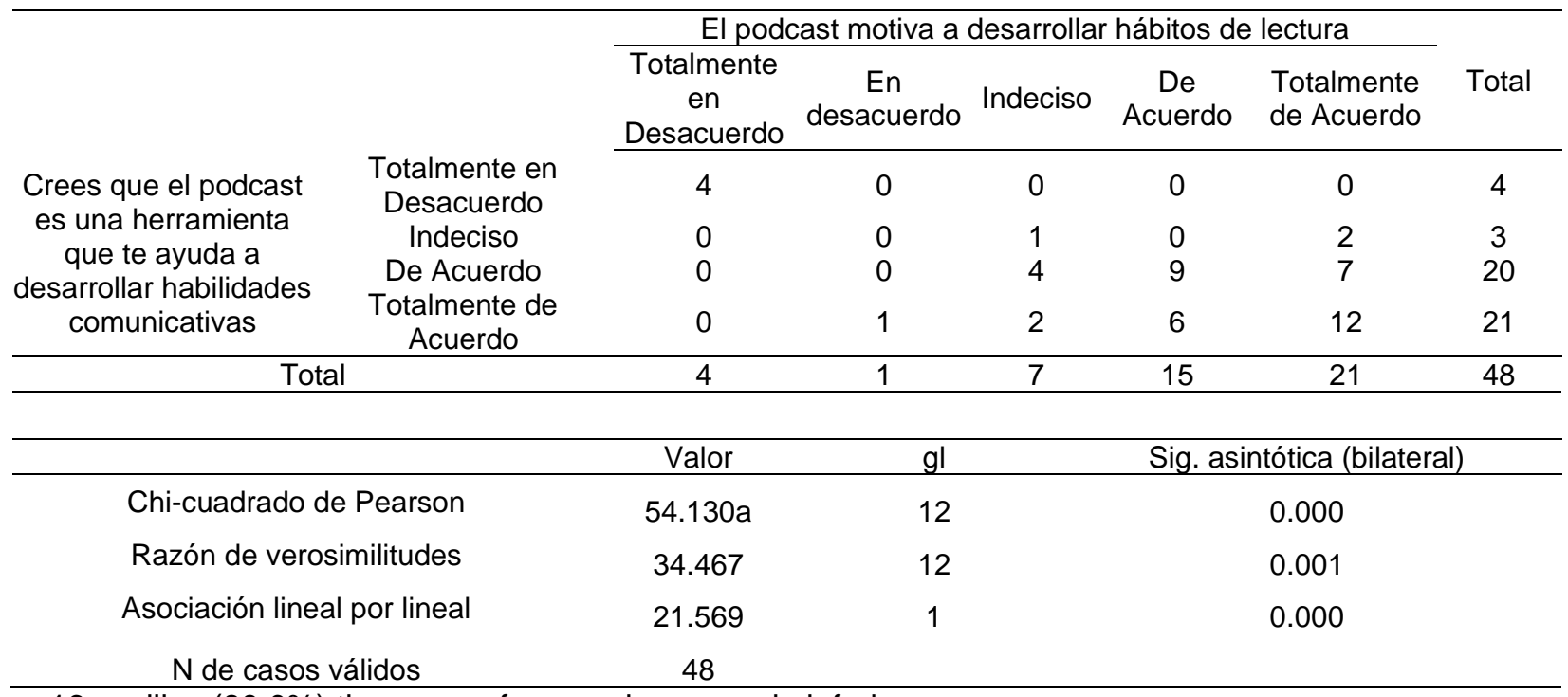

a. 16 casillas $(80.0 \%)$ tienen una frecuencia esperada inferior

a 5. La frecuencia mínima esperada es .06.

Fuente: Elaboración Propia.

En la tabla 3 se observa que en el cruce de variables obtiene una asignación bilateral, de 0.001 este resultado demuestra la relación entre las variables propuestas evidenciando que el podcast es útil para desarrollar hábitos de lectura y por ende permite desarrollar habilidades comunicativas, por lo concerniente la hipótesis es válida. 
EPISTEME KOINONIA

Revista Electrónica de Ciencias de la Educación, Humanidades, Artes y Bellas Artes

Año III. Vol III. N¹. Edición Especial. 2020

Hecho el depósito de Ley: FA2018000022

ISSN: 2665-0282

FUNDACIÓN KOINONIA (F.K).

Santa Ana de Coro, Venezuela

Iván Fernando Romero-Velásquez; Darwin Gabriel García-Herrera; Cristian Andrés Erazo-Álvarez Juan Carlos Erazo-Álvarez

\section{Tabla 4.}

Consideras que el podcast posee información desde el entorno académico * Estas conforme con el podcast como recurso de aprendizaje.

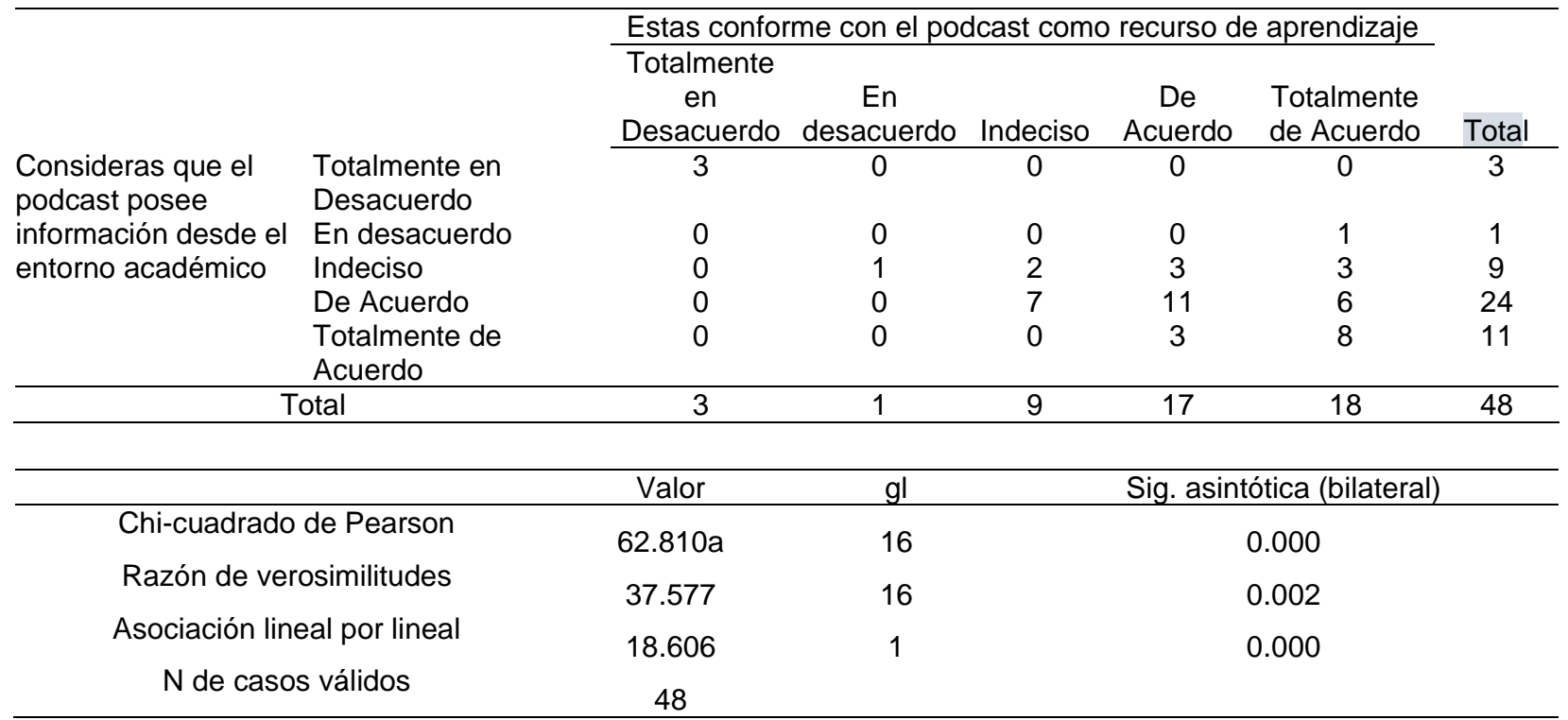

a. 23 casillas $(92.0 \%)$ tienen una frecuencia esperada inferior

a 5. La frecuencia mínima esperada es .02 .

Fuente: Elaboración Propia.

Mientas tanto que en la tabla 4 se puede evidenciar en el cruce de variables obtenemos una asignación bilateral de 0.002 este resultado está por debajo del rango normal por ende se puede comprobar la relación entre las variables existentes, demostrando que el podcast como recurso de aprendizaje tiene información desde el entorno académico y por ende permite desarrollar habilidades comunicativas, por lo concerniente la hipótesis es efectiva. 
Iván Fernando Romero-Velásquez; Darwin Gabriel García-Herrera; Cristian Andrés Erazo-Álvarez Juan Carlos Erazo-Álvarez

\section{Tabla 5.}

Crees que el podcast muestra información clara y contundente sobre los contenidos * EL podcast tiene un diseño atractivo para los oyentes.

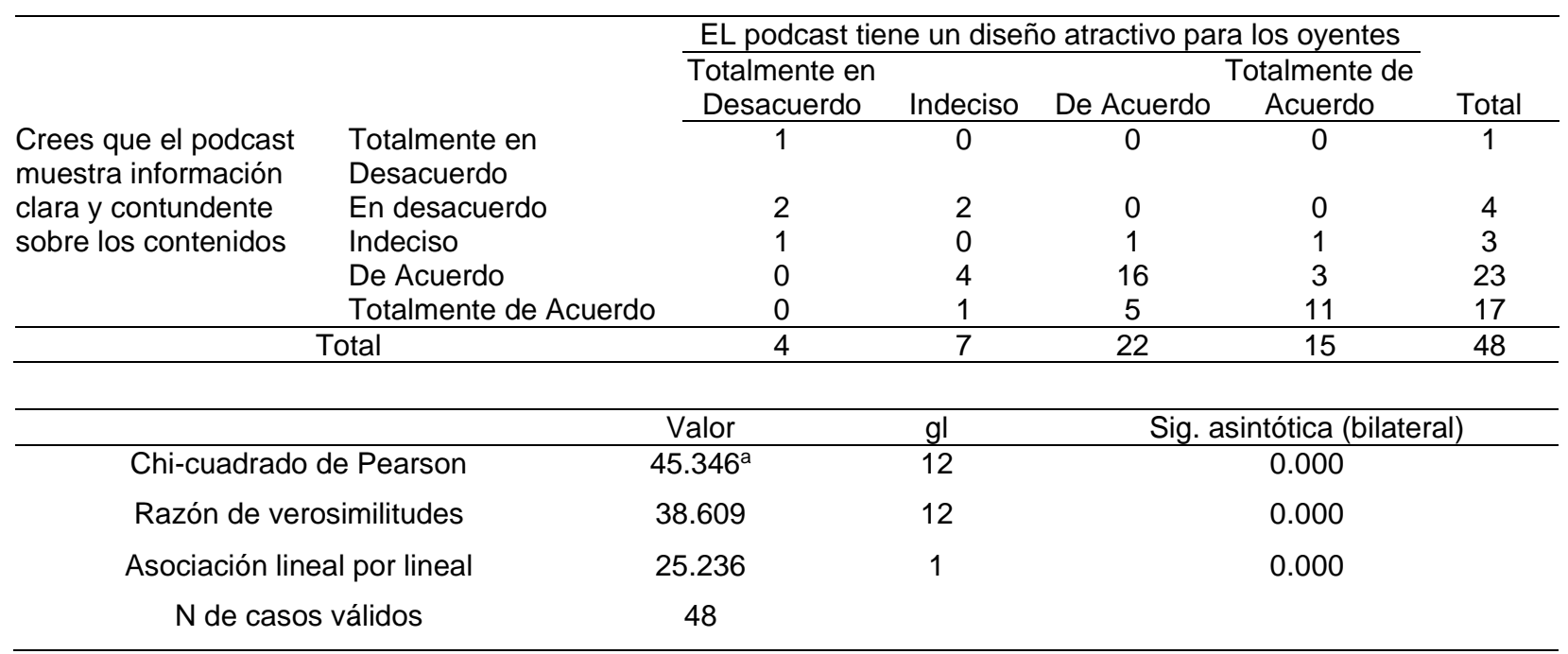

a. 16 casillas $(80.0 \%)$ tienen una frecuencia esperada inferior

a 5. La frecuencia mínima esperada es .08 .

Fuente: Elaboración Propia.

En la tabla 5 en el cruce de variables observa una asignación bilateral de 0.000 como resultado se muestra la relación entre las variables analizadas evidenciando que el diseño del podcast tiene información clara en sus contenidos por tal razón la hipótesis es válida.

Con la intensión de discutir el tema propuesto dentro de la investigación, a continuación, se describirá algunos criterios expuestos por otros autores; comenzare señalando que los elementos sonoros son la principal herramienta del podcast, gracias al sonido se puede elaborar verdaderos contenidos educativos, este recurso posee información objetiva y contrastada por ello se le considerar una herramienta eminentemente académica. 
Las tecnologías de la información y comunicación en la actualidad son utilizadas en la educación, estas herramientas son el apoyo en todos los niveles educativos, gracias que los docentes consideran a ese recurso un elemento necesario para desarrollar, potencializar y mejorar la pedagogía dentro del aula. (Martín-Álvarez, 2014). El uso del podcast en la educación permite a los estudiantes desarrollar sus propios sistemas de recreación, pero siempre apoyados en la tecnología, este recurso didáctico vincula una relación directa entre estudiantes y el docente, admite establecer procesos de aprendizaje sincrónicos como asincrónicos.

Gracias a las plataformas digitales se ha podido superar en cierta forma el acceso a la nueva movilidad de la información, esto se puede contrarrestar según los datos obtenidos en la investigación ejecutada a 48 estudiantes del nivel de bachillerato, donde se evidencia que el podcast agrega elementos que permiten que los usuarios desarrollen habilidades comunicativas, sus contenido conservan información desde el entorno académico y permite desarrollar nuevas experiencias de aprendizaje debido al diseño atractivo para el usuario. Como lo afirma (Aguiar-Freire, 2015) El podcast en la educación formal sirve para revelar los intereses de los estudiantes, es podcast tiene una modalidad de trabajo autónomo, ayuda a desarrollar aptitudes individuales, así como a sus deseos expresivos. 


\section{PROPUESTA}

En el proceso de enseñanza - aprendizaje se percibir claramente la estrecha relación entre la comunicación y la educación, pues; la comunicación es vital porque permite la transmisión o el intercambio de ideas entre las personas involucradas en el proceso formativo. A continuacion se presenta el Ciclo de planeacion del Podcast para desarrollar habilidades comunicativas.

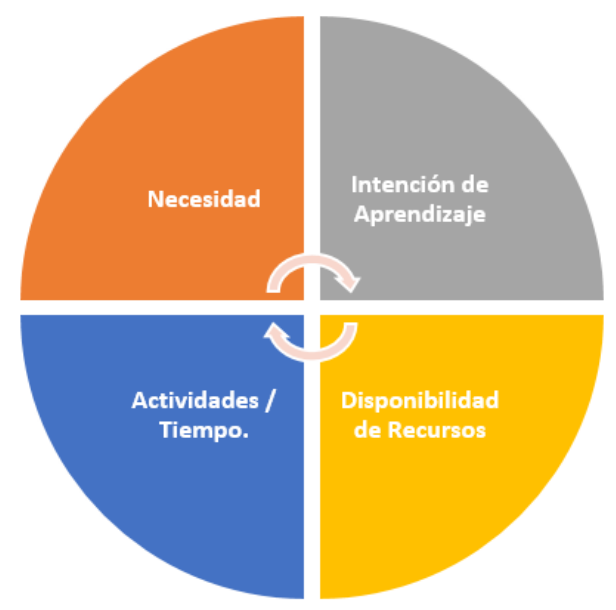

Figura 1. Ciclo de Planeación del podcast para desarrollar habilidades comunicativas. Fuente: Elaboración Propia.

Necesidad. - Es vital desarrollar la necesidad de comunicar de manera eficaz o de fortalecer las destrezas de lectura y escritura, puesto que; son elementos clave dentro del proceso de aprendizaje.

Intención de Aprendizaje. - Se sebe determinar con objetividad lo que el sujeto conocerá y aprenderá, implica tener claro lo que se le intenta trasmitir. En esta fase tiene como propósito identificar lo que el sujeto demostrará al término de su etapa de aprendizaje.

Disponibilidad de Recursos. - Se debe tomar en cuenta los medios tecnológicos como: el hardware, el software y los recursos humanos para la creación del guion, la producción, su distribución y evaluación del producto audiovisual. 
Actividades y Tiempo. - La elaboración de podcast favorece a la interacción de grupo, así como también al fortalecimiento de principios como el respeto y la responsabilidad en las actividades necesarias para la culminación de la producción.

El podcast como tecnología de la información y la comunicación desarrolla una gestión técnica en la educación, como medio de comunicación se transforma en el objeto de estudio, mientras que, como recurso educativo se convierte en material didáctico, el mismo que permite potencializar destrezas de lectoescritura; en este contexto, la comunicación se convierte en el componente necesario para desarrollar habilidades comunicativas en el individuo. Por tal razón como propuesta a la investigación realizada, se plantea el uso del podcast en el aula de clase y a la vez crear un blog para la recopilación de audio el mismo que servirá de repositorio para futuras consultas por parte de los estudiantes.

\section{CONCLUSIONES}

El podcast como Recurso Didáctico para Desarrollar Habilidades Comunicativas y por ende hemos logrado puntualizar temas como las habilidades Comunicativas, la falta de una buena comunicación en los salones de clase, el Uso del Podcast para desarrollar habilidades de comunicación, entre otras. Referirnos al podcast es, hablar de una de las herramientas de tendencia en la actualidad, los elementos técnicos que se requieren para editar, producir, escuchar o compartir un podcast es mínimo; es decir un micrófono, un celular, la computadora, un programa de audio libre permitirá que los usuarios sin ser peritos en tecnología consigan producir y compartir el contenido.

Tras los fundamentos obtenidos en esta investigación se llegan a la conclusión que; el podcast es útil para el proceso de enseñanza-aprendizaje puesto que su diseño es el mecanismo que permite desarrollar destrezas y competencias interdisciplinarias en cada uno de los usuarios. 
En concordancia con esto, el podcast también motiva y fortalece el hábito de la lectura, por ende, esta acción revitaliza el proceso de escritura, logrando así consolidar en el sujeto habilidades comunicativas. Por último es importante referirnos al sonido, este componente, es un elemento invisible, pero posee una fuerza poderosa al momento de comunicar o trasmitir un mensaje, el sonido moldea el cerebro y la manera de cómo aprenden; una historia o un contenido bien contado permite atraer la atención del oyente, por tal razón, en los datos obtenidos en la investigación, se demostró que el podcast conserva un diseño atractivo para sus consumidores, su género robustece el arte de narrar y a la vez incursiona en la oralidad digital elemento necesario para estimular el proceso de comunicar de manera eficaz dentro del aula o fuera de ella.

\section{FINANCIAMIENTO}

No monetario

\section{AGRADECIMIENTOS}

A la Unidad Educativa Particular Liceo Americano Católico de la Provincia del Azuay; por apoyar el desarrollo de la investigación.

\section{REFERENCIAS}

Aguiar Freire, E. (2015). Potenciais cooperativos do podcast escolar por uma perspectiva freinetiana. [Cooperative potentials of the school podcast from a Freinetian perspective]. Revista Brasileira de Educação, 20(63), 1033 - 1055

Aldana-Talero, S. (2012). El podcasts en la enseñanza de la lectoescritura. [The Podcasts for Teaching Reading and Writing]. Revista Virtual Universidad Católica del Norte(37), 9-26. 
Iván Fernando Romero-Velásquez; Darwin Gabriel García-Herrera; Cristian Andrés Erazo-Álvarez Juan Carlos Erazo-Álvarez

Boulahrouz-Lahmidi, M., Medir Huerta, R., \& Calabuig Serra, S. (2019). Tecnologías digitales y educación para el desarrollo sostenible. Un análisis de la producción científica. [Digital technologies and education for sustainable development. An analysis of scientific production]. Píxel-BIT Revista de Medios y Educación(54), 83105. https://doi.org/10.12795/pixelbit.2019.i54.05

Cañas-Betancur, D., \& Hernández-Sánchez, J. (2019). Comunicación asertiva en profesores: diagnóstico y propuesta educativa. [Assertive communication in teachers: diagnosis and educational proposal]. Praxis \& Saber, 10(24), 144 - 165. https://doi.org/10.19053/22160159.v10.n25.2019.8936

Cevallos-Mieles, O. (2016). Las habilidades comunicativas, una necesidad formativa del profesional del Siglo XXI. [Communication skills, a training need of the 21st century professional]. Yachana Revista Científica, 5(1), 130-137.

Cubero-Venegas, C. (2004). La disciplina en el aula: Reflexiones en torno a los procesos de comunicación. [Discipline in the classroom: Reflections on communication processes]. Revista Electrónica "Actualidades Investigativas en Educación", 4(2), 1-39.

Flores, D. (2016). La importancia e impacto de la lectura, redacción y pensamiento crítico en la educación superior. [The importance and impact of reading, writing and critical thinking in higher education]. Zona Próxima, 9(1); 128-135.

Gómez, J. (2016). La Comunicación. [The communication]. Salus, 20(3), 5-6.

Martín-Álvarez, J. (2014). Diseño de un proyecto de podcasting para la mejora de la expresión oral en la enseñanza de las lenguas. [Design of a podcasting project to improve oral expression in language learning]. Didáctica, Innovación y Multimedia (DIM) (28), 1-15.

Miranda-Calderón, L. (2011). Lenguaje: Algo más que un mecanismo para la comunicación. [Language: More than a Mechanism for Communication]. Revista Electrónica Educare, 15(1), 161-170.

Monsalve-Upegui, M. E., Franco-Velásquez, M. A., Monsalve-Ríos, M. A., BetancurTrujillo, V. L., \& Ramírez-Salazar, D. A. (2011). Desarrollo de las habilidades comunicativas en la escuela nueva. [Development of communicative skills in the new school]. Revista Educación y Pedagogía, 21(55), 189-210. 
Iván Fernando Romero-Velásquez; Darwin Gabriel García-Herrera; Cristian Andrés Erazo-Álvarez Juan Carlos Erazo-Álvarez

Moreno-Cazalla, L. (2017). Podium Podcast, cuando el podcasting tiene acento español. [Podium Podcast, when Podcasting has spanish accent]. Prisma Social: revista de investigación social(18), 334-364.

Quintana-Guerrero, B., Parra-Duque, C., \& Riaño-Peña, J. (2017). El podcast como herramienta para la innovación en espacios de comunicación universitarios. [The podcast as a tool for innovation in university communication spaces]. Anagramas, 15(30), 81-100. doi:10.22395/angr.v15n30a4

Reyzábal, M. (2012). Las Competencias Comunicativas y Lingüísticas, Clave para la Calidad Educativa. [Communicative and Linguistic Competences, Key to Educational Quality]. REICE. Revista Iberoamericana sobre Calidad, Eficacia y Cambio en Educación, 10(4), 1- 15.

Solano-Fernández, I., \& Sanchez-Vera, M. (2010). Aprendiendo En Cualquier Lugar: El Podcast. [Learning Anywhere: The Podcast Educational]. Pixel-Bit. Revista de Medios y Educación(36), 125-139. 